\title{
EMBARCACIONES FLUVIALES COMO MEDIO DE DISPERSIÓN DE Aedes aegypti HACIA ZONAS FRONTERIZAS DE LA AMAZONIA PERUANA
}

\author{
Carmen Sinti-Hesse ${ }^{1, a}$, Fabiola Díaz-Soria ${ }^{1, a}$, Wilma Casanova-Rojas ${ }^{2, b}$, Cristiam Carey-Ángeles ${ }^{2, c}$, \\ Rodil Tello-Espinoza ${ }^{3, d}$, José Espinoza ${ }^{4, e}$, Karine Zevallos ${ }^{2, f}$
}

\begin{abstract}
RESUMEN
Objetivos. Evaluar el riesgo entomológico de Aedes aegypti durante el recorrido fluvial en embarcaciones con rutas fronterizas de Loreto. Materiales y métodos. La población de estudio fueron los mosquitos presentes en tres embarcaciones con rutas fronterizas en Loreto. El riesgo entomológico de Aedes se determinó mediante ovitrampas, revisión de criaderos, colecta e identificación taxonómica de mosquitos adultos. Resultados. El riesgo entomológico varió según trayecto y época. Se identificó un riesgo entomológico medio a muy alto en época de creciente y en la ruta de ida hacia las zonas fronterizas. La población vectorial predominante en época de vaciante fue Mansonia $s p$. $(74,8 \%)$, Culex sp. $(12,8 \%)$ y Aedes aegypti $(0,4 \%)$; en creciente predominó Culex sp. $(45,1 \%)$, Mansonia sp. $(26,8 \%)$ y Aedes aegypti (19,7\%). En ningún caso se evidenció Aedes albopictus. Conclusiones. Existe riesgo entomológico de moderado a alto durante la etapa de creciente en las embarcaciones fluviales que viajan desde lquitos hacia las zonas fronterizas de Loreto. Nuestros resultados evidencian que las embarcaciones fluviales son un medio de dispersión de Aedes aegypti.
\end{abstract}

Palabras clave: Mosquitos vectores; Aedes; Control sanitario de puertos y embarcaciones; Áreas fronterizas (fuente: DeCS BIREME).

\section{RIVER BOATS AS A MEANS OF EXPANSION OF Aedes aegypti TOWARDS BORDER ZONES OF THE PERUVIAN AMAZON}

\begin{abstract}
Objectives. To assess the entomological risk of Aedes aegypti in boats traversing border river routes in Loreto. Materials and Methods. The study population consisted of mosquitoes present in three boats covering border routes in Loreto. The entomological risk of Aedes was determined through ovitraps, the inspection of breeding sites, and the collection and taxonomic identification of adult mosquitoes. Results. The entomological risk varied according to the route and the season. A medium to very high entomological risk was identified in the high-water season and on the outward route to the border areas. The predominant vector population in the low-water season was Mansonia sp. (74.8\%), Culex sp. (12.8\%), and Aedes aegypti (0.4\%); in the high-water season, Culex sp. (45.1\%), Mansonia sp. (26.8\%), and Aedes aegypti $(19.7 \%)$. In no case did we find Aedes albopictus. Conclusions. There is moderate to high entomological risk during the high-water season in riverboats traveling from lquitos to the border areas of Loreto. Our results show that river boats are a means of expansion of Aedes aegypti.
\end{abstract}

Keywords: Mosquito vectors; Aedes; Sanitary control of harbors and crafts; Border areas (source: MeSH NLM).

\footnotetext{
Centro de Investigación en Enfermedades Tropicales «Hugo Pesce-Maxime Kuckynski», Instituto Nacional de Salud, Iquitos, Perú Facultad de Medicina Humana, Universidad Nacional de la Amazonia Peruana, Iquitos, Perú

Vicerrectorado de Investigación, Universidad Nacional de la Amazonia Peruana, Iquitos, Perú

Facultad de Ingeniería Económica, Estadística y Ciencias Sociales de la Universidad Nacional de Ingeniería, Lima, Perú

Bióloga; ${ }^{b}$ licenciada en Enfermería, magister en Salud Pública; ${ }^{c}$ médico, magister en Salud Pública; ${ }^{d}$ ingeniero forestal, doctor en Ciencias Ambientales; ${ }^{\mathrm{e}}$ licenciado en Estadística, maestría en Ciencias; ${ }^{\mathrm{f}}$ médico, doctor en Salud Pública

Recibido: 23/05/2019 Aprobado: 31/07/2019 En línea: 27/08/2019
}

Citar como: Sinti-Hesse C, Díaz-Soria F, Casanova-Rojas W, Carey-Angeles C, Tello-Espinoza R, Espinoza J, et al. Embarcaciones fluviales como medio de dispersión de Aedes aegypti hacia zonas fronterizas de la Amazonia peruana. Rev Peru Med Exp Salud Publica. 2019;36(3):392-9. doi: http://dx.doi.org/10.17843/rpmesp.2019.363.4558. 


\section{INTRODUCCIÓN}

Aedes aegypti es el vector responsable de la dispersión de enfermedades como dengue, chikungunya y zika, las cuales tienen una creciente importancia a nivel mundial por su carga de enfermedad como por el potencial epidémico ${ }^{(1-3)}$. La prevención de estas enfermedades, a través de una vacuna y otras intervenciones innovadoras son prometedoras pero aún no están disponibles en el corto plazo ${ }^{(4,5)}$. Por lo tanto, la vigilancia vectorial continúa siendo la principal herramienta para la prevención y control de Aedes aegypti (6).

A nivel mundial se ha demostrado que la expansión de las actividades económicas como el transporte terrestre o fluvial, desde áreas urbanas hacia áreas rurales ha llevado a la diseminación de Aedes aegypti, incrementando el riesgo en el área rural (7-9). Los determinantes socioeconómicos como la pobreza y la falta de servicios básicos sumado al incremento vectorial han convertido a estas zonas en un blanco de brotes epidémicos ${ }^{(7,10)}$.

Se ha reportado en Manaos (Brasil) y Leticia (Colombia) la presencia de Aedes aegypti y Aedes albopictus (7,9) y en la región Loreto (Perú) la mayoría de sus distritos tienen la presencia de Aedes aegypti (11,12). En este contexto epidemiológico, el desplazamiento de embarcaciones fluviales podrían propagar Aedes desde ciudades pobladas hacia zonas remotas con baja densidad vectorial $(8,9)$. El incremento de la población de mosquitos en regiones fronterizas podría tener graves consecuencias en la población indígena; podría colapsar los servicios de salud e incrementar la morbimortalidad por dengue, chikungunya y zika debido a la escasa capacidad resolutiva de los centros de salud de zonas rurales.

La Dirección Ejecutiva de Salud Ambiental de Loreto (DESA Loreto), quien es la autoridad sanitaria local, realiza la vigilancia entomológica del Aedes aegypti en la comunidad, a nivel intra y peridomiciliario. La vigilancia se realiza por un profesional de salud, quien visita e inspecciona los recipientes con agua y colecta las larvas presentes ${ }^{(11)}$. Esta práctica presenta obstáculos como la escasez de recursos humanos, limitados recursos económicos para actividades extramurales, demora en el tiempo de recolección y procesamiento de la información epidemiológica ${ }^{(13)}$. Por lo cual, desde el 2015 se introdujo el uso de ovitrampas como parte de la vigilancia entomológica de Aedes aegypti (14,15).

Las embarcaciones fluviales son un medio de transporte popular en las zonas de selva, y tienen características propicias para la reproducción de Aedes. Albergan espacios con sombra y humedad, transportan agua en diferentes recipientes y se desplazan con carga y pasajeros a través de ciudades pobladas y rurales ${ }^{(9)}$. Por lo cual, consideramos imperante conocer el riesgo entomológico, presencia y abundancia de Aedes en estas embarcaciones

\section{MENSAJES CLAVE}

Motivación para realizar el estudio. Conocer el riesgo entomológico in situ de Aedes en embarcaciones fluviales con rutas fronterizas.

Principales hallazgos. Las embarcaciones fluviales son un medio de dispersión de Aedes aegypti. El riesgo entomológico de Aedes aegypti fue bajo en vaciante y de retorno hacia Iquitos; de medio a muy alto en creciente y de ida hacia zonas fronterizas. La población vectorial hallada fue: Mansonia sp., Culex sp. y Aedes aegypti en vaciante y Culex sp., Mansonia sp. y Aedes aegypti. en creciente. No se encontró Aedes albopictus.

Implicancias. Existe un riesgo entomológico de moderado a alto en época de creciente en embarcaciones fluviales que viajan desde Iquitos hacia las zonas fronterizas de Loreto.

y demostrar que pueden ser vehículos de dispersión de Aedes en la cuenca amazónica. Asimismo, consideramos necesario evaluar el uso de la ovitrampas para detectar poblaciones de Aedes aegypti ${ }^{(16)}$ en este nuevo escenario, no descrito en publicaciones previas.

El objetivo de nuestro estudio fue evaluar el riesgo entomológico de Aedes aegypti durante el recorrido fluvial de embarcaciones con rutas fronterizas de Loreto.

\section{MATERIALES Y MÉTODOS}

\section{DISEÑO Y UBICACIÓN}

Se realizó un estudio descriptivo longitudinal en la región Loreto en la Amazonia peruana. Loreto es una región endémica y presenta la mayor frecuencia de casos de dengue, zika y malaria en Perú. El estudio se desarrolló entre los meses de setiembre a noviembre del 2016 (época de creciente) y de mayo a julio del 2017 (época de vaciante) en embarcaciones fluviales con rutas fronterizas hacia Caballococha ( $3^{\circ} 54^{\prime} 29.44^{\prime \prime}-S$ y $70^{\circ} 30^{\prime} 58.02$ "-O y $77 \mathrm{msnm}$ a orillas del río Amazonas); Santa Rosa ( $4^{\circ} 13^{\prime} 10.41^{\prime \prime}-S$ y $69^{\circ} 57^{\prime} 59.64^{\prime \prime}-O$ y 100 msnm a orillas del río Amazonas, frontera con Colombia y Brasil), y El Estrecho $\left(2^{\circ} 27^{\prime} 1.6^{\prime \prime}-\mathrm{S}\right.$ y $72^{\circ} 40^{\prime} 4.4^{\prime \prime}-\mathrm{O}$ y $110 \mathrm{msnm}$ a orillas del río Putumayo).

Se seleccionaron embarcaciones de tres pisos que transporten pasajeros y carga, y que tengan un largo promedio de 50 metros. 13 embarcaciones hacia Caballococha y Santa Rosa y cuatro embarcaciones hacia El Estrecho cumplieron estos criterios. Se seleccionó al azar una embarcación por trayecto.

\section{RECOLECCIÓN DE DATOS}

Para la ejecución del proyecto, se tuvo la autorización del director de la DIRESA Loreto (Oficio N ${ }^{\circ}$ 483-2015GRL-DRSL/30.09-INVESTIGACIÓN). Se firmaron las 
autorizaciones para ingresar a las embarcaciones y permitir que un miembro del equipo de investigación viaje en las rutas y realice la vigilancia entomológica durante el trayecto. Se realizaron dos viajes, uno en creciente (caracterizada por el aumento del caudal de los ríos) y otro en vaciante (caracterizada por la disminución del caudal de los ríos) ${ }^{(17)}$. Se analizaron tres diferentes embarcaciones con ruta a Caballococha, Santa Rosa y El Estrecho. La vigilancia entomológica se realizó durante el trayecto de ida y de vuelta; en total dos revisiones para cada embarcación, para la colecta de huevos, larvas/pupas y mosquitos (Tabla 1) (Figura 1).

\section{RECOLECCIÓN DE LA INFORMACIÓN}

La información se recolectó utilizando un instrumento diseñado y digitalizado en una tableta electrónica, utilizando el paquete Open Data Kit con captura de datos, almacenados localmente y luego transferidos a la nube (Google Drive) con una copia de seguridad disponible off-line y luego transferidos al Centro de Investigación en Enfermedades Tropicales (CIETROP) del Instituto Nacional de Salud (INS). Las tiras de papel con huevos fueron captadas con cámaras HAD-CCD de 20,1 megapixeles, almacenadas en las memorias y procesadas con el programa Imagen J para tiras hasta 20 huevos, para un número mayor de huevos se utilizó el Estereomicroscópio SZX16. Se revisaron todos los criaderos potenciales para larvas y pupas. Se colectaron mosquitos adultos con aspiradores Prokopack. Se tomaron las coordenadas con un GPS Garmin Etrex 20X.

\section{DETERMINACIÓN DEL RIESGO ENTOMOLÓGICO}

El riesgo entomológico fue definido como la presencia y abundancia de los insectos vectores de alguna enfermedad en un lugar determinado ${ }^{(18)}$. Definido a través de los siguientes indicadores: índice de positividad de ovitrampas (IPO), índice de densidad de huevos (IDH) ${ }^{(14)}$ e índice de adultos $(\mathrm{IA})^{(13)}$.

La inspección de huevos en ovitrampas: las ovitrampas son depósitos plásticos que en el exterior contienen un rótulo de identificación. Como sustrato se usa papel toalla y como atrayente se usa la infusión de pasto. El criterio utilizado para la colocación de ovitrampas se basó en lo descrito en la norma técnica para viviendas ${ }^{(11)}$. Cada piso de la embarcación se consideró una vivienda y se colocaron ovitrampas cada 50 metros, teniendo en cuenta que el radio de vuelo del mosquito es de 50 a 100 metros; al colocar las ovitrampas dentro de las embarcaciones, se eligieron zonas oscuras, libres de flujos de viento y sin exposición a lluvias ${ }^{(14)}$. Cuando el papel era positivo se recogía y se reemplazaba por otro. Se procedía a capturar la imagen, tomar la ubicación geográfica del lugar (19) (Tabla 2) y guardarlo en una bolsa plástica rotulada.

Tabla 1. Características de las embarcaciones según estación y trayecto

\begin{tabular}{|c|c|c|c|c|c|c|}
\hline \multirow{2}{*}{ Características } & \multicolumn{3}{|c|}{ Creciente } & \multicolumn{3}{|c|}{ Vaciante } \\
\hline & Caballococha & Santa Rosa & El Estrecho & Caballococha & Santa Rosa & El Estrecho \\
\hline Hora partida & $20: 41: 00$ & $22: 43: 00$ & 04:00:00 & $21: 10: 00$ & $22: 10: 00$ & $06: 50: 00$ \\
\hline Número de pasajeros & 145 & 143 & 40 & 134 & 111 & 50 \\
\hline Número de pasajeros por piso* & $\begin{array}{l}\text { P1: } 5 \\
\text { P2: } 70 \\
\text { P3: } 70\end{array}$ & $\begin{array}{l}\text { P1: } 6 \\
\text { P2: } 80 \\
\text { P3: } 56\end{array}$ & $\begin{array}{l}\text { P1: } 8 \\
\text { P2: } 12 \\
\text { P3: } 20\end{array}$ & $\begin{array}{l}\text { P1: } 7 \\
\text { P2: } 72 \\
\text { P3: } 55\end{array}$ & $\begin{array}{l}\text { P1: } 7 \\
\text { P2: } 56 \\
\text { P3: } 47\end{array}$ & $\begin{array}{l}\text { P1: } 10 \\
\text { P2: } 20 \\
\text { P3: } 20\end{array}$ \\
\hline Número de pasajeros por habitación & 2 & 3 & 2 & 2 & 2 & 2 \\
\hline Número de recipientes inspeccionados & 23 & 30 & 27 & 14 & 38 & 21 \\
\hline Transportan agua potable & No & No & $\mathrm{Si}$ & No & No & $\mathrm{Si}$ \\
\hline Número de pisos & 3 & 3 & 3 & 3 & 4 & 3 \\
\hline Número de ambientes por piso & $\begin{array}{l}\text { P1: } 4 \\
\text { P2: } 4 \\
\text { P3: } 5\end{array}$ & $\begin{array}{l}\text { P1: } 4 \\
\text { P2: } 4 \\
\text { P3: } 4\end{array}$ & $\begin{array}{l}\text { P1: } 4 \\
\text { P2: } 4 \\
\text { P3: } 3\end{array}$ & $\begin{array}{l}\text { P1: } 4 \\
\text { P2: } 4 \\
\text { P3: } 5\end{array}$ & $\begin{array}{l}\text { P1: } 4 \\
\text { P2: } 4 \\
\text { P3: } 4\end{array}$ & $\begin{array}{l}\text { P1: } 4 \\
\text { P2: } 4 \\
\text { P3: } 3\end{array}$ \\
\hline Material de pared & Metal & Metal & Metal & Metal & Metal & Metal \\
\hline Material de techo & Madera/metal & Metal & Metal & Madera/metal & Metal & Metal \\
\hline Material de piso & Metal & Metal & Metal & Metal & Metal & Metal \\
\hline Material de ventanas & $\begin{array}{c}\text { Metal, } \\
\text { vidrio } \\
\text { y mica }\end{array}$ & $\begin{array}{c}\text { Metal, } \\
\text { vidrio } \\
\text { y mica }\end{array}$ & $\begin{array}{c}\text { Malla } \\
\text { contra } \\
\text { insectos }\end{array}$ & $\begin{array}{c}\text { Metal, } \\
\text { vidrio } \\
\text { y mica }\end{array}$ & $\begin{array}{c}\text { Metal, } \\
\text { vidrio } \\
\text { y mica }\end{array}$ & $\begin{array}{c}\text { Malla } \\
\text { contra } \\
\text { insectos }\end{array}$ \\
\hline Cocina & Gas & Gas & Gas & Gas & Gas & Gas \\
\hline Fuente de agua para cocinar & Rio & Rio & Tratada & Rio & Rio & Tratada \\
\hline Eliminación de desechos & Bolsas & Balde & Balde y bolsas & Bolsas & Bolsas & Bolsas \\
\hline
\end{tabular}

$\mathrm{P} 1$ : piso uno, P2: piso dos, P3: piso tres 

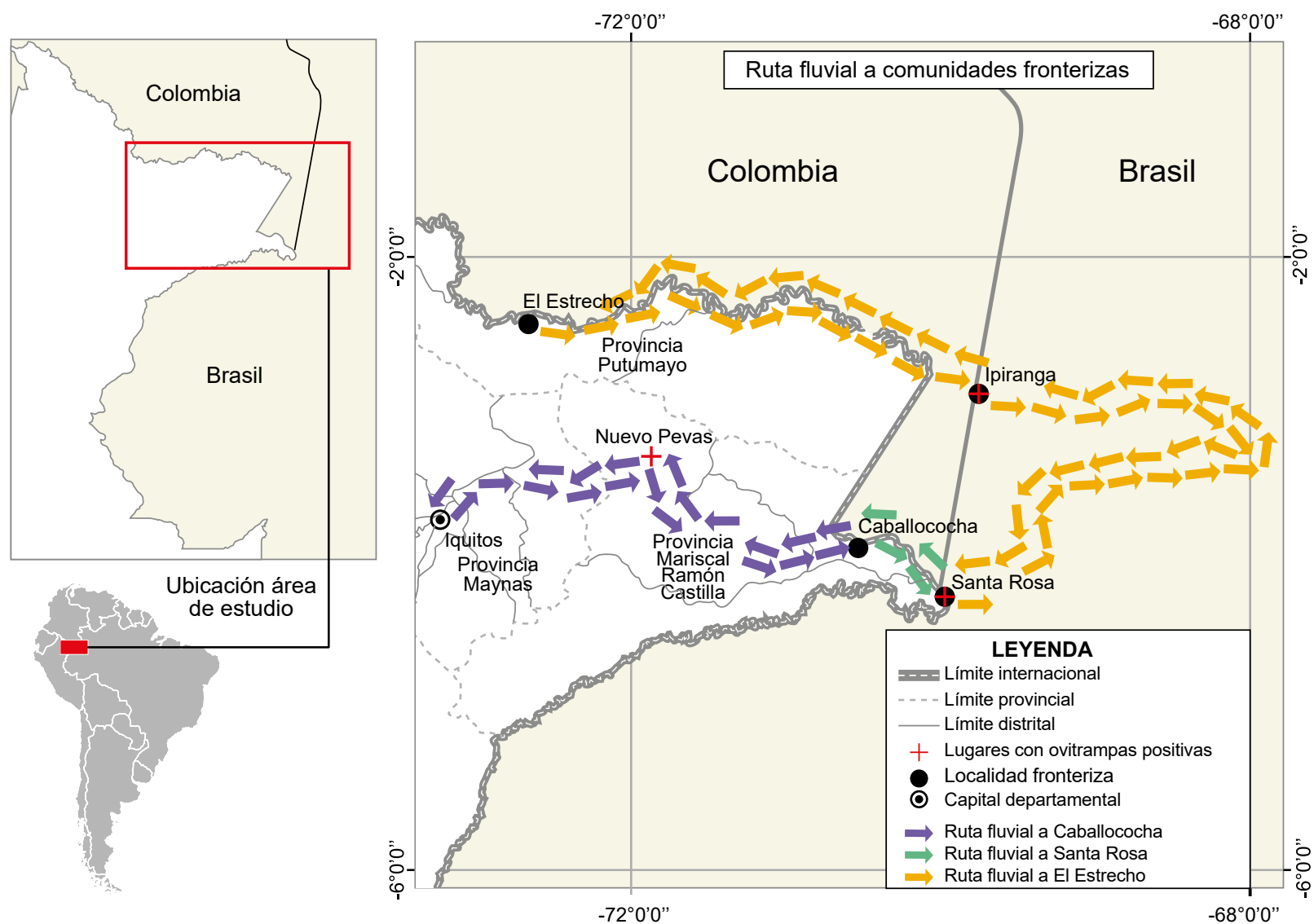

Figura 1. Mapa del recorrido de las embarcaciones hacia las localidades fronterizas (trayecto de ida y vuelta)

La inspección de larvas/pupas: se buscó en cada piso de la embarcación recipientes con agua que pueden contener larvas o pupas, de acuerdo al formato de criaderos utilizado por la DESA Loreto ${ }^{(11,20)}$.
La captura de adultos: los mosquitos adultos fueron capturados con aspiradores Prokopack a lo largo de las paredes, pisos y ambientes, coincidiendo con su horario de alimentación (8.00 a 10.00 h y 17.00 a 19.00 h) ${ }^{(21) .}$

Tabla 2. Localización geoespacial de las ovitrampas en las embarcaciones fluviales

\begin{tabular}{|c|c|c|c|c|c|}
\hline \multicolumn{2}{|c|}{ Georeferenciación } & \multirow{2}{*}{ Localidades } & \multirow{2}{*}{$\begin{array}{c}\text { Tiempo de permanencia } \\
\text { de las barcazas }\end{array}$} & \multirow{2}{*}{ Habitantes } & \multirow{2}{*}{$\begin{array}{c}\text { Positividad de } \\
\text { ovitrampas }\end{array}$} \\
\hline Latitud & Longitud & & & & \\
\hline-3.487269 & -72.190797 & Apayacu & $1 \mathrm{~h}$ & 285 & - \\
\hline-3.322942 & -71.862146 & Pevas & $2 \mathrm{~h}$ & 3788 & + \\
\hline-3.371125 & -71.776023 & Nuevo Pevas & $30 \mathrm{~min}$ & 482 & - \\
\hline-3.758134 & -71.624055 & Cochiquina & $2 \mathrm{~h}$ & 1041 & - \\
\hline-3.806504 & -71.573452 & San Antonio & $1 \mathrm{~h}$ & 709 & - \\
\hline-3.863192 & -71.352887 & San Isidro & $1 \mathrm{~h}$ & 525 & - \\
\hline-4.017102 & -71.100457 & San Pablo & $2 \mathrm{~h}$ & 3137 & - \\
\hline-3.927604 & -70.778901 & Chimbote & $2 \mathrm{~h}$ & 344 & - \\
\hline-3.905087 & -70.517707 & Caballococha & $2 \mathrm{~h}$ & 9051 & - \\
\hline-4.219842 & -69.955005 & Santa Rosa & $2 \mathrm{~h}$ & 970 & + \\
\hline-4.3524 & -70.040455 & Islandia & 1 día & 1692 & - \\
\hline-2.148826 & -71.753218 & Ipiranga & $1 \mathrm{~h}$ & 456 & + \\
\hline-2.893369 & -69.734015 & Tarapaca & $1 \mathrm{~h}$ & 289 & - \\
\hline-2.539835 & -70.429 & Huapapa & $1 \mathrm{~h}$ & 355 & - \\
\hline-2.385124 & -71.181284 & Remanso & $1 \mathrm{~h}$ & 412 & - \\
\hline-2.445288 & -72.668377 & El Estrecho & 1 semana & 3056 & - \\
\hline
\end{tabular}


Posteriormente, se les expuso a acetato de etilo para conservación y transporte en placas petri hasta ser llevados al laboratorio del CIETROP para identificarlos.

\section{ANÁLISIS DE LOS DATOS}

El riesgo entomológico se midió a partir de indicadores entomológicos como el índice de densidad de Aedes a partir del índice de densidad de huevos (IDH) que resulta de la división del número de huevos encontrados entre número de ovitrampas positivas, clasificado como riesgo bajo: 1 a $<5$ huevos, riesgo medio: 5 a $<20$ huevos, riesgo alto: 20 a $<40$ huevos y riesgo muy alto: $>40$ huevos ${ }^{(14)}$. El Índice de Adultos (IA) se refiere al número de mosquitos; clasificado como bajo riesgo de 1 a 3 mosquitos y alto riesgo: >3 mosquitos ${ }^{(13)}$. El índice de positividad de Aedes a partir del índice de positividad de ovitrampas (IPO) que resulta de la división del número de ovitrampas positivas entre el número de ovitrampas expuestas $\times 100$, clasificado como riesgo bajo: $<5 \%$, riesgo medio: $5 \%$ a $<20 \%$, riesgo alto: $20 \%$ a $<40 \%$ y riesgo muy alto: $>40 \%{ }^{(14)}$. Las poblaciones vectoriales colectadas fueron identificadas con claves taxonómicas ${ }^{(22)}$.

Los procedimientos estadísticos para el procesamiento de los datos fueron expresados a través de frecuencias y porcentajes. Se utilizó el paquete estadístico SPSS versión 21.

\section{ASPECTOS ÉTICOS}

Esta investigación tuvo un riesgo mínimo para las personas de las embarcaciones fluviales participantes. Las inspecciones entomológicas realizadas en el estudio fueron idénticas a las llevadas a cabo por la DESA Loreto. No se generó un mayor riesgo de transmisión de estas enfermedades para los participantes más allá de la experimentada al vivir en una zona endémica. Los resultados de este estudio serán de gran beneficio para las actividades de control local de vectores. Todas las consideraciones éticas fueron revisadas y aprobadas por el Comité de Investigación de Ética de Animales del INS con resolución directoral $\mathrm{N}^{\circ} 580-2016-O G I T T-O P E / I N S$.

\section{RESULTADOS}

\section{ÍNDICE DE DENSIDAD Y POSITIVIDAD DE Aedes sp.}

En la ruta a Caballococha, los índices de IDH e IA fueron de $0 \%$ en la ida y en la vuelta tanto en creciente como en vaciante. En la ruta a Santa Rosa en época de creciente se encontraron de 26 a 66 huevos, mientras que en vaciante no se encontró ningún huevo. En la ruta a El Estrecho, en época de creciente se encontraron hasta siete huevos. Las interpretaciones de estos hallazgos se detallan en la Tabla 3. En ninguna de las rutas se logró evidenciar huevos de Aedes albopictus.

En la ruta a Caballococha el IPO fue bajo en la ida y en la vuelta, tanto en creciente como en vaciante. En la ruta a Santa Rosa el IPO fue de $14 \%$ en creciente en el trayecto de ida y vuelta y en vaciante fue de $0 \%$. En la ruta a El Estrecho, el IPO fue de $20 \%$ tanto en creciente como en vaciante en el trayecto de ida y de $0 \%$ en el trayecto de vuelta (Tabla 3). En ninguna de las rutas se logró evidenciar Aedes albopictus en las ovitrampas.

Se inspeccionaron los recipientes que podrían albergar larvas y/o pupas de Aedes, encontramos en mayor proporción, tanto en creciente como en vaciante, los servibles (platos, sartenes, porta platos), baldes y ollas y en menor proporción los tanques, cilindros y llantas. No se encontró ningún criadero positivo.

\section{POBLACIONES VECTORIALES}

La población vectorial encontrada fue Aedes aegypti, Aediomia, Anopheles nuneztovari, Anopheles peryasui, Anopheles trianulatus, Coquelletidia, Culex melanoconium, Culex sp. y Mansonia sp. La población vectorial predominante en época de vaciante fue Mansonia sp. $(74,8 \%)$ que transmite filariasis linfática, Culex sp. $(12,8 \%)$ que transmite encefalitis, oropuche, filariasis, virus del Nilo y Aedes aegypti $(0,4 \%)$ transmite dengue, zika y chikungunya ${ }^{(23,24)}$, en creciente predominó Culex sp. (45,1\%), Mansonia sp. (26,8\%) y Aedes aegypti (19,7\%). En la Tabla 4, se describen las poblaciones vectoriales según trayecto y época.

Tabla 3. Índice de densidad e índice de positividad de Aedes según estación y trayecto

\begin{tabular}{|c|c|c|c|c|c|c|c|c|c|c|c|c|}
\hline \multirow{3}{*}{ Trayecto } & \multicolumn{4}{|c|}{ IDH } & \multicolumn{4}{|c|}{ IPO } & \multicolumn{4}{|c|}{ IA } \\
\hline & \multicolumn{2}{|c|}{ Creciente } & \multicolumn{2}{|c|}{ Vaciante } & \multicolumn{2}{|c|}{ Creciente } & \multicolumn{2}{|c|}{ Vaciante } & \multicolumn{2}{|c|}{ Creciente } & \multicolumn{2}{|c|}{ Vaciante } \\
\hline & Ida & Vuelta & Ida & Vuelta & Ida & Vuelta & Ida & Vuelta & Ida & Vuelta & Ida & Vuelta \\
\hline Caballococha & Bajo & Bajo & Bajo & Bajo & Bajo & Bajo & Bajo & Bajo & Bajo & Bajo & Bajo & Bajo \\
\hline Santa Rosa & Muy alto & Alto & Bajo & Bajo & Medio & Medio & Bajo & Bajo & Bajo & Bajo & Bajo & Bajo \\
\hline El Estrecho & Medio & Bajo & Bajo & Bajo & Alto & Bajo & Alto & Bajo & Medio & Bajo & Bajo & Bajo \\
\hline
\end{tabular}

IDH: índice de densidad de huevos, IPO: índice de positividad de ovitrampas, IA: índice de adultos 
Tabla 4. Distribución de vectores en embarcaciones fluviales según estación y trayecto

\begin{tabular}{|c|c|c|c|c|c|c|c|c|c|c|c|c|c|c|c|c|}
\hline \multirow{3}{*}{ Variable } & \multicolumn{4}{|c|}{ Caballococha } & \multicolumn{4}{|c|}{ Santa Rosa } & \multicolumn{4}{|c|}{ El Estrecho } & \multicolumn{4}{|c|}{ Total } \\
\hline & \multicolumn{2}{|c|}{ Creciente } & \multicolumn{2}{|c|}{ Vaciante } & \multicolumn{2}{|c|}{ Creciente } & \multicolumn{2}{|c|}{ Vaciante } & \multicolumn{2}{|c|}{ Creciente } & \multicolumn{2}{|c|}{ Vaciante } & \multicolumn{2}{|c|}{ Creciente } & \multicolumn{2}{|c|}{ Vaciante } \\
\hline & $\mathbf{n}$ & $\%$ & $\mathbf{n}$ & $\%$ & $\mathbf{n}$ & $\%$ & $\mathbf{n}$ & $\%$ & $\mathbf{n}$ & $\%$ & $\mathbf{n}$ & $\%$ & $\mathbf{n}$ & $\%$ & $\mathbf{n}$ & $\%$ \\
\hline \multicolumn{17}{|l|}{ Especie de mosquitos } \\
\hline Aedes aegypti & 0 & 0,0 & 0 & 0,0 & 1 & 8,3 & 0 & 0,0 & 13 & 40,6 & 1 & 7,7 & 14 & 19,7 & 1 & 0,4 \\
\hline Aediomia & 1 & 3,7 & 15 & 37,5 & 0 & 0,0 & 12 & 5,2 & 1 & 3,1 & 0 & 0,0 & 2 & 2,8 & 27 & 9,5 \\
\hline Anopheles nuneztovari & 0 & 0,0 & 0 & 0,0 & 0 & 0,0 & 2 & 0,9 & 0 & 0,0 & 0 & 0,0 & 0 & 0,0 & 2 & 0,7 \\
\hline Anopheles peryasui & 0 & 0,0 & 0 & 0,0 & 0 & 0,0 & 0 & 0,0 & 1 & 3,1 & 0 & 0,0 & 1 & 1,4 & 0 & 0,0 \\
\hline Anopheles trianulatus & 0 & 0,0 & 0 & 0,0 & 0 & 0,0 & 1 & 0,4 & 0 & 0,0 & 0 & 0,0 & 0 & 0,0 & 1 & 0,4 \\
\hline Coquelletidia & 0 & 0,0 & 0 & 0,0 & 0 & 0,0 & 3 & 1,3 & 2 & 6,3 & 0 & 0,0 & 2 & 2,8 & 3 & 1,1 \\
\hline Culex melanoconium & 0 & 0,0 & 0 & 0,0 & 1 & 8,3 & 1 & 0,4 & 0 & 0,0 & 0 & 0,0 & 1 & 1,4 & 1 & 0,4 \\
\hline Culex sp. & 16 & 59,3 & 7 & 17,5 & 7 & 58,3 & 17 & 7,5 & 9 & 28,1 & 12 & 92,3 & 32 & 45,1 & 36 & 12,7 \\
\hline Mansonia sp. & 10 & 37,0 & 18 & 45,0 & 3 & 25,0 & 193 & 84,3 & 6 & 18,8 & 0 & 0,0 & 19 & 26,8 & 211 & 74,8 \\
\hline \multicolumn{17}{|l|}{ Sexo de los mosquitos } \\
\hline Hembra & 21 & 77,8 & 33 & 82,5 & 10 & 83,3 & 214 & 93,4 & 22 & 68,7 & 11 & 84,6 & 53 & 74,6 & 258 & 91,5 \\
\hline Macho & 6 & 22,2 & 7 & 17,5 & 2 & 16,7 & 15 & 6,6 & 10 & 31,3 & 2 & 15,4 & 18 & 25,4 & 24 & 8,5 \\
\hline \multicolumn{17}{|l|}{ Piso de embarcación } \\
\hline P1 (nivel del piso) & 8 & 29,6 & 19 & 47,5 & 8 & 66,6 & 40 & 17,5 & 22 & 68,8 & 2 & 15,3 & 38 & 53,5 & 61 & 21,6 \\
\hline P2 (2 m altura) & 6 & 22,2 & 8 & 20,0 & 2 & 16,7 & 65 & 28,4 & 5 & 15,6 & 5 & 38,5 & 13 & 18,3 & 78 & 27,7 \\
\hline P3 (4,5 m altura) & 13 & 48,2 & 13 & 32,5 & 2 & 16,7 & 124 & 54,1 & 5 & 15,6 & 6 & 46,2 & 20 & 28,2 & 143 & 50,7 \\
\hline
\end{tabular}

P1: piso uno, P2: piso dos, P3: piso tres

\section{DISCUSIÓN}

Se demostró que en épocas de creciente existe riesgo entomológico de Aedes aegypti en las embarcaciones fluviales que se desplazan desde poblaciones endémicas urbanas hacia áreas no endémicas rurales. Por lo tanto, podemos considerar a las embarcaciones fluviales como un medio de dispersión de Aedes aegypti.

El principal medio de transporte en la Amazonia son las embarcaciones fluviales, estas tienen diferentes características según la cantidad de pasajeros, tipo de carga y distancia a recorrer. Diferentes locaciones han demostrado ser reservorios de Aedes aegypti, sin embargo, estos estudios fueron realizados en ciudades, casas o puertos y no en el recorrido fluvial de una embarcación ${ }^{(8,9)}$. Durante el desplazamiento de las embarcaciones fluviales no se evidenciaron criaderos positivos, sin embargo, se identificaron criaderos potenciales como servibles (platos, sartenes, porta platos), baldes y ollas y en menor proporción los tanques, cilindros y llantas. A pesar de no haber encontrado formas inmaduras en los potenciales criaderos y una menor población adulta de Aedes aegypti con respecto a los otros vectores; el presente estudio demuestra que las embarcaciones fluviales constituyen medios de dispersión del Aedes aegypti hacia lugares menos poblados con condiciones apropiadas para su establecimiento.

Evaluar el riesgo entomológico durante el recorrido permitió conocer la dinámica operativa de las embarcaciones in situ e identificar blancos específicos para futuras intervenciones en salud. Por ejemplo, conocemos ahora que la ruta hacia la localidad de El Estrecho, por ser la de mayor duración, transporta suministro de agua en contenedores sin protección y fue en este trayecto donde se evidenció un mayor IDH e IPO.

En uno de los periodos del estudio (setiembre a noviembre de 2016) se desarrolló un brote de zika en la ciudad de Iquitos que se dispersó rápidamente a la provincia de Ramón Castilla y a zonas rurales de la provincia de Maynas; zonas por donde se desplazaron las embarcaciones fluviales que fueron evaluadas en nuestro estudio. Aunque el diseño de nuestra investigación no nos permite afirmar que las embarcaciones fluviales dispersaron zika, es posible que hayan contribuido en esta epidemia, tal como se ha descrito en otras epidemias a nivel mundial ${ }^{(3,25)}$.

Nuestros resultados sobre Aedes aegypti refuerza lo previamente publicado sobre su capacidad de seguir un patrón de expansión asociado al crecimiento de ciudades, nuevas carreteras y a lo largo de los ríos ${ }^{(8)}$. La capacidad de Aedes aegypti para infestar zonas urbanas en crecimiento, puede verse acelerado por los medios de transporte que pueden transportar a estos vectores hacia las zonas urbanas como lquitos, principalmente si retornan de zonas fronterizas con la Amazonia brasileña y colombiana, con presencia de Aedes albopictus y brotes de chikungunya.

No se colectaron huevos, pupas o formas adultas de Aedes albopictus, coincidiendo con lo reportado por un estudio local en una región fronteriza de Loreto ${ }^{(26)}$. Posiblemente los factores que impiden el establecimiento de Aedes 
albopictus podrían ser el río Amazonas actuando como barrera geográfica, y que los productos transportados en las embarcaciones de Leticia y Tabatinga a Santa Rosa no funcionan como criaderos para este vector. Asimismo, no es posible confirmar la ausencia del vector cuando la vigilancia realizada fue menor de un año ${ }^{(11)}$

EI IDH y el IA fue mayor en creciente y cuando las embarcaciones partían de lquitos, una ciudad endémica con alta densidad poblacional ${ }^{(15,27)}$, la densidad poblacional y las precipitaciones pluviales coinciden con un incremento de la presencia del vector en todos sus estadios ${ }^{(8)}$

En relación a la población vectorial, Aedes aegypti no fue predominante y sólo fue mayor en creciente. El mosquito con mayor población fue Mansonia sp. seguido de Culex; evidenciando la riqueza de especies de mosquitos y su distribución estacional. A pesar de haber encontrado diferentes vectores en formas adultas, durante la inspección de ovitrampas sólo se evidenció huevo de Aedes y no de otros vectores ${ }^{(28)}$. La emergencia y reemergencia de algunas enfermedades transmitidas por mosquitos es uno de los aspectos más relevantes en el continente americano, por lo que conocer la fauna de mosquitos, así como su distribución es imperante para un adecuado control vectorial y establecer estrategias para evitar la propagación de enfermedades transmisibles ${ }^{(29)}$.

Las ovitrampas permitieron determinar un IDH muy alto en una ruta, demostrando su utilidad y sensibilidad en transportes fluviales, lo cual confirma que se pueden utilizar en nuevos lugares y condiciones de vigilancia o estudios de investigación ${ }^{(12,30)}$.

Algunas limitaciones se refieren al diseño que no permitió evaluar el grado de paridad de los mosquitos, realizar pruebas moleculares y no incluir evaluación de febriles durante el recorrido fluvial. Asimismo, notamos que ante nuestra presencia se evidenció mayores acciones de limpieza dentro de las embarcaciones, lo cual puede haber reducido la población vectorial. A pesar de estas limitaciones, nuestro estudio ha demostrado que las embarcaciones fluviales son un medio de dispersión de Aedes aegypti hacia zonas rurales de la Amazonia peruana. Por lo cual, las autoridades locales de salud deben reforzar la vigilancia vectorial en las embarcaciones fluviales.

Agradecimientos: Al personal de los puestos de salud, colegios y base militares que facilitaron el uso de sus instalaciones. A la unidad de investigación de la Universidad Nacional de la Amazonia Peruana. A la Dra. Amy Morrison, catedrática honorable de la Universidad Nacional de la Amazonía Peruana. A Ana Claudia Ríos Araujo y Valeria Pinedo Torres por su valioso apoyo dentro de las embarcaciones en la ejecución del proyecto. A Miguel A. Farfán García, Víctor A. Torres Ocmin, Ricarte R. Ruiz Chávez y Rosa Liz Castro Bardales, personal de apoyo del CIETROP.

Contribuciones de autoría: CSH participó en la concepción, diseño del manuscrito, recolección, análisis, interpretación de los datos, redacción y revisión crítica importante del contenido del manuscrito. FDS participó en la concepción, recolección, análisis, interpretación de los datos, redacción y revisión crítica importante del contenido del manuscrito. WCR participó en la redacción y revisión crítica importante del contenido del manuscrito. CCA participó en la redacción y revisión crítica importante del contenido del manuscrito. RTE participó en el análisis e interpretación de los datos, redacción y revisión crítica importante del contenido del manuscrito. JE participó en el análisis e interpretación de los datos, redacción y revisión crítica importante del contenido del manuscrito. KZ participó en el diseño del manuscrito, análisis, interpretación de los datos, redacción y revisión crítica importante del contenido del manuscrito.

Fuente de financiamiento: Fondos de investigación de la Universidad Nacional de la Amazonia Peruana. Resolución Rectoral $N^{\circ}$ 0772-2015-UNAP.

Conflictos de interés: Declaramos no tener conflictos de interés

\section{REFERENCIAS BIBLIOGRÁFICAS}

1. World Health Organization. Global strategy for dengue prevention and control, 2012-2020. [Internet]. Geneva: World Health Organization; 2012 [citado 3 de mayo de 2019]. Disponible en: http://apps.who.int/iris/bitstre $\mathrm{am} / 10665 / 75303 / 1 / 9789241504034$ eng.pdf

2. Morens D, Fauci A. Chikungunya at the door-Déjà vu all over again? N Engl J Med. 2014;371(10):885-7.

3. Núñez E, Vásquez M, Beltrán-Luque $B$, Padgett D. Virus Zika en Centroamérica y sus complicaciones. Acta Méd Peru. 2016;33(1):42-9.

4. Slifka M. Vaccine-Mediated Immunity Against Dengue and the Potential for Long-Term Protection Against Disease.
Front Immunol. 2014;5:195. doi: 10.3389/fimmu.2014.00195.

5. McNaughton D, Huong-Duong T. Designing a Community Engagement Framework for a New Dengue Control Method: A Case Study from Central Vietnam. PLoS Negl Trop Dis. 2014;8(5):e2794. doi: 10.1371/journal. pntd.0002794.

6. Hernández-Ávila J, Rodríguez $\mathrm{M}$, Santos-Luna R, Sánchez-Castañeda V, Román-Pérez S, Ríos-Salgado V, et al. Nation-Wide, Web-Based, Geographic Information System for the Integrated Surveillance and Control of Dengue Fever in Mexico. PLoS One. 2013;8(8):e70231. doi: 10.1371/journal.pone.0070231.
7. Barbosa MG, Fé NF, Jesus RD, Rodriguez IC, Monteiro WM, Mourão MP, et al. Aedes aegypti e fauna associada em área rural de Manaus, na Amazônia brasileira. Rev Soc Bras Med Trop. 2009;42(2):213-6.

8. Guagliardo S, Barboza J, Morrison A, Astete H, Vazquez-Prokopec G, Kitron U. Patterns of Geographic Expansion of Aedes aegypti in the Peruvian Amazon. Barrera R, editor. PLoS Negl Trop Dis. 2014;8(8):e3033. doi:10.1371/journal. pntd.0003033.

9. Guagliardo S, Morrison A, Barboza J, Requena E, Astete H, Vazquez-Prokopec G, et al. River Boats Contribute to the Regional Spread of the Dengue Vector Aedes aegypti in the Peruvian Amazon. 
PLoSNegl TropDis.2015;9(4):e0003648. doi:10.1371/journal.pntd.0003648.

10. Harrington L, Fleisher A, Ruiz-Moreno D, Vermeylen F, Wa C, Poulson R, et al. Heterogeneous Feeding Patterns of the Dengue Vector, Aedes aegypti, on Individual Human Hosts in Rural Thailand. PLoS Negl Trop Dis. 2014;8(8):e3048. doi:10.1371/journal. pntd.0003048.

11. Dirección General de Salud Ambiental. Ministerio de Salud del Perú. Norma Técnica de Salud para la Implementación de la Vigilancia y Control de Aedes aegypti, Vector del Dengue en el Territorio Nacional. Primera Edición [Internet]. Lima: MINSA; 2011 [citado 3 mayo de 2019]. Disponible en: http://www.digesa.minsa.gob.pe/ publicaciones/descargas/NORMA\%20 Aedes\%20aegypti_DSB.pdf

12. Cabezas C, Fiestas V, García-Mendoza M, Palomino M, Mamani E, Donaires F. Dengue en el Perú: a un cuarto de siglo de su reemergencia. Rev Peru Med Exp Salud Publica. 2015;32(1):146-56.

13. Villaseca P, Cáceres A, Linares N. Eficacia de las ovitrampas para la detección rápida de Aedes aegypti en Chanchamayo (Junín) y Pucallpa (Ucayali), Perú. Bol - Inst Nac Salud. 2006;12(11-12):321-2.

14. Ministerio de Salud del Perú, Dirección General de Intervenciones Estratégicas en Salud Pública. Protocolo Sanitario de urgencia para el reforzamiento de la Vigilancia Entomológica del vector Aedes aegypti mediante el uso de ovitrampas para Establecimientos de Salud. Primera Edición [Internet]. Lima: MINSA; 2016 [citado 3 mayo de 2019]. Disponible en: http://bvs.minsa. gob.pe/local/MINSA/3817.pdf

15. Veiga-Acioli R. Uso de ovitrampas como herramienta para el monitoreo de poblaciones deuA Aedes spp en el barrio de Recife [Tesis de Maestría]. Recife, Brasil: Fundación Oswaldo Cruz; 2006.

16. Domínguez-Galera M. Evaluación de ovitrampas como sistema de vigilancia entomológica en sitios públicos de Chetumal, Quintana Roo [Tesis Doctoral]. Nuevo León, México: Universidad Autónoma de Nuevo León; 2010.
17. Sistema de Información del Agua y las Cuencas de la Amazonía Peruana [Internet]. SIAGUA; 2019 [citado 3 mayo de 2019]. Características de las aguas amazónicas. Disponible en: http://www. siaguaamazonia.org.pe/caracteristicas_ hidricos.html

18. Norma oficial mexicana, para la vigilancia epidemiológica, prevención y control de las enfermedades transmitidas por vector. NOM-032-SSA2-2010 de 01/06/2011. Disponible en: http://www.cenaprece. salud.gob. $\mathrm{mx} /$ programas/interior/ vectores/descargas/pdf/nom_032_ ssa2_2010_norma_petv.pdf

19. Instituto Nacional de Estadística e Informática. Capítulo 19 Departamento Loreto. Principales Indicadores Departamentales 2009 - 2015 [Internet]. Lima: INEI; 2019 [citado 3 mayo de 2019]. Disponible en: https://www.inei.gob.pe/ media/MenuRecursivo/publicaciones digitales/Est/Lib1340/cuadros/cap18.pdf

20. Durand Velazco S, Fiestas Solórzano V, Sihuincha Maldonado M, Chávez Lencinas C, Vásquez Vela V, et al. Impacto de la epidemia de dengue con un nuevo linaje del Denv-2 genotipo americano / asiático en la demanda de servicios del hospital de apoyo de Iquitos "César Garayar García”. Rev Peru Med Exp Salud Publica. 2011;28(1):157.

21. Organización Panamericana de la Salud. Dengue: Información general. [Internet]. Washington DC: PAHO; 2019. Disponible en: https://www.paho.org/ hq/index.php?option=com_content\&vi ew=article\&id=4493:2010-informaciongeneral-dengue $\&$ Itemid $=40232 \&$ lang $=e s$

22. Consoli R, deOliveira R. Principais mosquitos de importância sanitária no Brasil. Rio de Janeiro, RJ: Editora FIOCRUZ; $1994.225 \mathrm{p}$.

23. Swiger SL. Los mosquitos y las enfermedades que transmiten [Internet]. Texas: The Texas A\&M Agrilife Extension Service; 2019 [citado 12 marzo de 2019]. Disponible en: https://cdn-ext.agnet. tamu.edu/wp-content/uploads/2016/09/ Los-mosquitos-y-las-enfermedades-quetransmiten.pdf

24. Organización Panamericana de la Salud. Diez enfermedades transmitidas por vectores que ponen en riesgo a la población de las Américas [Internet]. Washington DC: PAHO; 2019. Disponible en: https://www.paho.org/hq/index. php?option $=$ com_content $\&$ view $=$ art icle\&id=9438:2014-10-vector-bornediseases-that-put-population-americas-atrisk\&Itemid $=135 \&$ lang $=$ es

25. Centro Nacional de Epidemiología, Dirección y Control de Enfermedades. Enfermedad por virus Zika - Perú 2016 y 2017 [Internet]. Lima: Ministerio de Salud del Perú; 2017 [citado 3 mayo de 2019]. Disponible en: http://www. dge.gob.pe/portal/docs/vigilancia/ sala/2017/SE08/zika.pdf

26. Rios-Araujo A. Positividad y Riesgo Entomológico de Aedes albopictus (Skuse, 1894) relacionado con los factores ambientales en la localidad de Santa Rosa, Loreto - Perú [Tesis]. Iquitos: Facultad de Ciencias Biológicas. Universidad Nacional de la Amazonía Peruana; 2015.

27. Rubio-Palis Y, Pérez-Ybarra LM, InfanteRuíz M, Comach G, Urdaneta-Márquez L. Influencia de las variables climáticas en la casuística de dengue y la abundancia de Aedes aegypti (Diptera: Culicidae) en Maracay, Venezuela. Boletin Malariol Salud Ambient. 2011;51(2):145-58.

28. Peraza Cuesta I, Pérez Castillo M, Mendizábal Alcalá ME, Valdés Miró V, Leyva Silva M, Marquetti Fernández M del C. Richness and distribution of Culicidae species in the province of Havana, Cuba. Rev Cubana Med Trop. 2015;67(2):270-8.

29. Valdés-Miró V, Reyes-Arencibia M, Marquetti-Fernández M, GonzalesBroche R. Riqueza de especies de mosquitos, distribución y sitios de cría en el Municipio Boyeros. Rev Cubana Med Trop. 2013;65 (1):131-6.

30. Kilpatrick J, Tonn R, Jatanasen S. Evaluation of ultra-low-volume insecticide dispensing systems for use in single-engined aircraft and their effectiveness against Aedes aegypti populations in SouthEast Asia. Bull World Health Organ. 1970;42(1):1.

Correspondencia: Carmen Sinti Hesse Dirección: Calle Guardia Republicana $N^{\circ}$ 190 - San Juan Bautista. Iquitos, Perú. Teléfono: 956428149

Correo electrónico: carsinhes@gmail.com 